\title{
Cosmic structure as the quantum interference of a coherent dark wave
}

\author{
Hsi-Yu Schive ${ }^{1}$, Tzihong Chiueh ${ }^{1,2 \star}$ and Tom Broadhurst ${ }^{3,4}$
}

The conventional cold-particle interpretation of dark matter (known as 'cold dark matter', or CDM) still lacks laboratory support and struggles with the basic properties of common dwarf galaxies, which have surprisingly uniform central masses and shallow density profiles ${ }^{1-5}$. In contrast, galaxies predicted by CDM extend to much lower masses, with steeper, singular profiles ${ }^{6-9}$. This tension motivates cold, wavelike dark matter ( $\psi D M)$ composed of a non-relativistic Bose-Einstein condensate, so the uncertainty principle counters gravity below a Jeans scale ${ }^{10-12}$. Here we achieve cosmological simulations of this quantum state at unprecedentedly high resolution capable of resolving dwarf galaxies, with only one free parameter, $m_{B}$, the boson mass. We demonstrate the large-scale structure is indistinguishable from CDM, as desired, but differs radically inside galaxies where quantum interference forms solitonic cores surrounded by extended haloes of fluctuating density granules. These results allow us to determine $m_{B}=\left(8.0_{-2.0}^{+1.8}\right) \times 10^{-23} \mathrm{eV}$ using stellar phase-space distributions in dwarf spheroidal galaxies. Denser, more massive solitons are predicted for Milky Way sized galaxies, providing a substantial seed to help explain early spheroid formation. The onset of galaxy formation is substantially delayed relative to $C D M$, appearing at redshift $z \lesssim 13$ in our simulations.

Standard, thermally generated dark matter remains firmly undetected in laboratory searches for weakly interacting massive particles (WIMPs; ref. 13). Non-thermal bosonic fields, particularly scalar fields, provide another well-motivated class of dark matter, formed in a non-relativistic, low-momentum state as a cold Bose-Einstein condensate (BEC), and increasingly motivated by extensions of the Standard Model of particle physics and to the mechanism driving the universal expansion ${ }^{14}$. The field in this context can be described by a coherent wave function $\psi$ with an interference pattern determining the distribution of dark matter, which we term $\psi \mathrm{DM}$. Axions are long-standing CDM candidates of this form, and higher-dimensional theories motivate an 'axiverse', where a discrete mass spectrum of axion-like particles spans many decades, possibly affecting cosmic structure ${ }^{15}$.

The distribution of $\psi \mathrm{DM}$ mimics particle CDM on large scales $^{16,17}$, and hence distinguishing between CDM and cold, wavelike $\psi \mathrm{DM}$ is best made on small scales owing to the additional quantum stress ${ }^{10-12,17}$. Dwarf spheroidal $(\mathrm{d} S p h)$ galaxies are the smallest and most common class of galaxy with internal motions dominated by dark matter. Their basic properties are very hard to explain with standard CDM, including the surprising uniformity of their central masses, $M(<300 \mathrm{pc}) \simeq 10^{7} M_{\odot}$, where $M_{\odot}$ is the solar mass, and shallow density profiles ${ }^{1-5}$. In contrast, galaxies
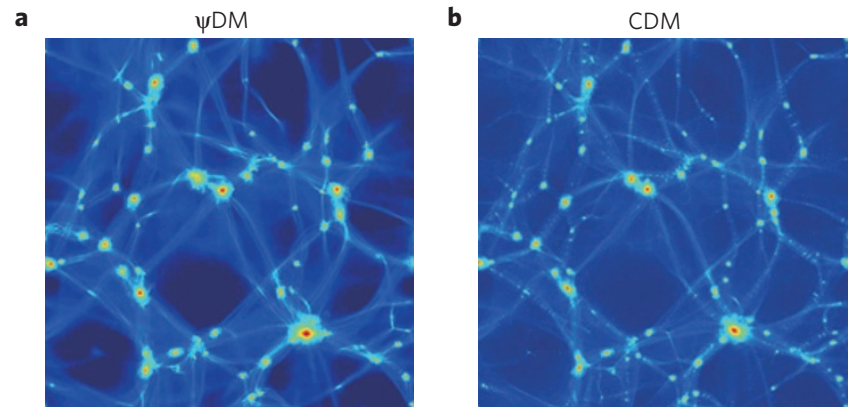

Figure 1 | Comparison of cosmological large-scale structures formed by standard CDM and by wavelike dark matter, $\psi D$ DM. a, Structure created by evolving a single coherent wave function for $\Lambda \psi D M$ calculated on adaptive-mesh-refinement grids. b, Structure simulated with a standard $\Lambda$ CDM N-body code GADGET-2 (ref. 34) for the same cosmological parameters, with the high- $k$ modes of the linear power spectrum intentionally suppressed in a way similar to the $\psi \mathrm{DM}$ model to highlight the comparison of large-scale features. This comparison clearly demonstrates that the large-scale distribution of filaments and voids is indistinguishable between our model and $\Lambda C D M$ (which has been successful in describing the observed large-scale structure). $\psi D M$ arises from the low-momentum state of the condensate so that it is equivalent to collisionless CDM well above the Jeans scale.

predicted by CDM extend to much lower masses, well below the observed dwarf galaxies, with steeper, singular mass profiles ${ }^{6-9}$. Adjustments to standard CDM addressing these difficulties consider particle collisions ${ }^{18}$, or warm dark matter (WDM; ref. 19). WDM can be tuned to suppress small-scale structures, but does not provide large enough flat cores $^{20}$. Collisional CDM can be adjusted to generate flat cores, but cannot suppress low-mass galaxies without resorting to other baryonic physics ${ }^{21}$. Better agreement is expected for $\psi \mathrm{DM}$ because the uncertainty principle counters gravity below a Jeans scale, simultaneously suppressing small-scale structures and limiting the central density of collapsed haloes ${ }^{10-12}$.

Detailed examination of structure formation with $\psi \mathrm{DM}$ is therefore highly desirable, but, unlike the extensive $\mathrm{N}$-body investigation of standard CDM, no sufficiently high resolution simulations of $\psi \mathrm{DM}$ have been attempted. The wave mechanics of $\psi \mathrm{DM}$ can be described by Schrödinger's equation, coupled to gravity by means of Poisson's equation ${ }^{16}$ with negligible microscopic selfinteraction. The dynamics here differs from collisionless particle CDM by a new form of stress tensor from quantum uncertainty,

\footnotetext{
${ }^{1}$ Department of Physics, National Taiwan University, No. 1, Sec. 4, Roosevelt Road, Taipei, 10617, Taiwan, ${ }^{2}$ Center for Theoretical Sciences, National Taiwan University, Taipei 10617, Taiwan, ${ }^{3}$ Department of Theoretical Physics, University of the Basque Country UPV/EHU, E-48080 Bilbao, Spain, ${ }^{4}$ Ikerbasque, Basque Foundation for Science, E-48011 Bilbao, Spain. *e-mail: chiuehth@phys.ntu.edu.tw
} 


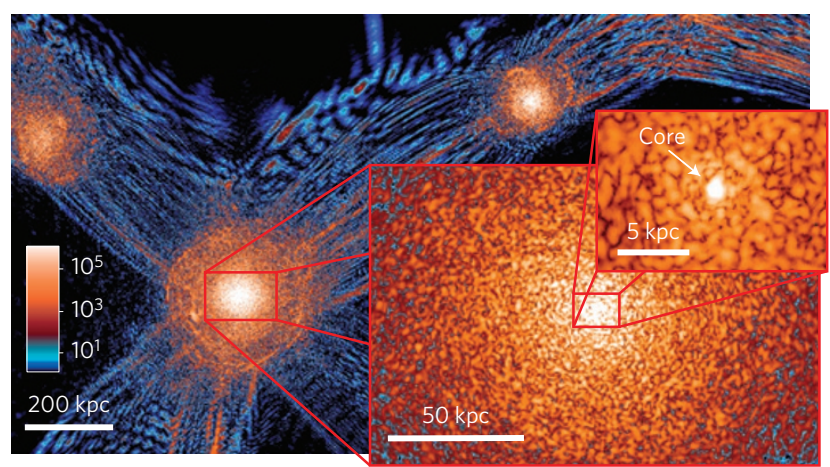

Figure 2 | A slice of the density field of the $\psi \mathrm{DM}$ simulation on various scales at $z=0.1$. This scaled sequence (each of thickness $60 \mathrm{pc}$ ) shows how quantum interference patterns can be clearly seen everywhere from the large-scale filaments, tangential fringes near the virial boundaries, to the granular structure inside the haloes. Distinct solitonic cores with radii $\sim 0.3-1.6 \mathrm{kpc}$ are found within collapsed haloes (which have virial masses $\left.M_{\text {vir }} \sim 10^{9 \sim 10^{11}} M_{\odot}\right)$. The density shown here spans over nine orders of magnitude, from $10^{-1}$ to $10^{8}$ (normalized to the cosmic mean density). The colour map scales logarithmically, with cyan corresponding to density $\lesssim 10$.

giving rise to a co-moving Jeans length, $\lambda_{\mathrm{J}} \propto(1+z)^{1 / 4} m_{\mathrm{B}}^{-1 / 2}$, during the matter-dominated epoch ${ }^{17}$. The insensitivity of $\lambda_{\mathrm{I}}$ to redshift, $z$, generates a sharp cutoff mass below which structures are suppressed. Cosmological simulations in this context turn out to be much more challenging than standard N-body simulations, as the highest frequency oscillations, $\omega$, given approximately by the matter wave dispersion relation, $\omega \propto m_{\mathrm{B}}^{-1} \lambda^{-2}$, where $\lambda$ is the wavelength, occur on the smallest scales, requiring very fine temporal resolution even for moderate spatial resolution (Supplementary Fig. 1). In this work, we optimize an adaptive-mesh-refinement (AMR) scheme, with graphic processing unit acceleration, improving performance by almost two orders of magnitude ${ }^{22}$ (see Supplementary Section 1 for details).

Figure 1 demonstrates that despite the completely different calculations employed, the pattern of filaments and voids generated by a conventional $\mathrm{N}$-body particle $\Lambda \mathrm{CDM}$ simulation is remarkably indistinguishable from the wavelike $\Lambda \psi \mathrm{DM}$ for the same linear power spectrum (Supplementary Fig. 3). Here $\Lambda$ represents the cosmological constant. This agreement is desirable given the success of standard $\triangle \mathrm{CDM}$ in describing the statistics of large-scale structure. To examine the wave nature that distinguishes $\psi \mathrm{DM}$ from $\mathrm{CDM}$ on small scales, we re-simulate with a very high maximum resolution of $60 \mathrm{pc}$ for a $2 \mathrm{Mpc}$ co-moving box, so that the densest objects formed of $\gtrsim 300 \mathrm{pc}$ size are well resolved with $\sim 10^{3}$ grids. A slice through this box is shown in Fig. 2, revealing fine interference fringes defining long filaments, with tangential fringes near the boundaries of virialized objects, where the de Broglie wavelengths depend on the local velocity of matter. An unexpected feature of our $\psi \mathrm{DM}$ simulations is the generation of prominent dense coherent standing waves of dark matter in the centre of every gravitational bound object, forming a flat core with a sharp boundary (Figs 2 and 3). These dark matter cores grow as material is accreted and are surrounded by virialized haloes of material with fine-scale, large-amplitude cellular interference, which continuously fluctuate in density and velocity, generating quantum and turbulent pressure support against gravity.

The central density profiles of all our collapsed cores fit well the stable soliton solution of the Schrödinger-Poisson equation, as shown in Fig. 3 (see also Supplementary Section 2 and Figs 2 and 4). On the other hand, except for the lightest halo, which has just formed and is not yet virialized, the outer profiles of other haloes possess a steepening logarithmic slope, similar to the Navarro-Frenk-White

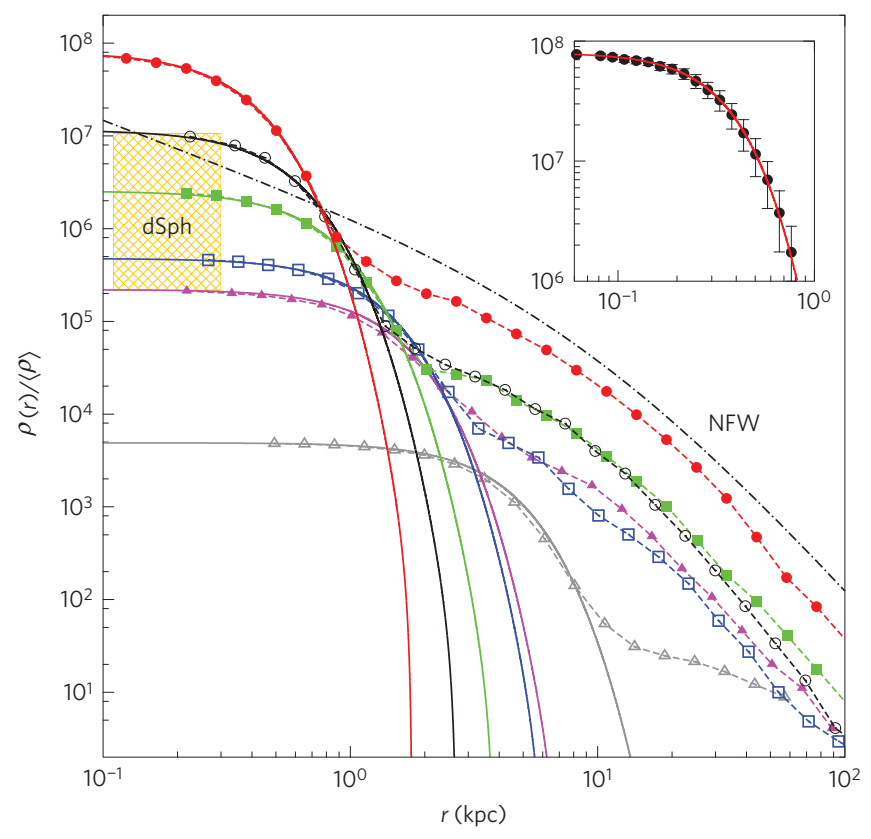

Figure 3 | Radial density profiles of haloes formed in the $\psi D M$ model.

Dashed lines with various symbols show six examples of the halo profiles normalized to the cosmic mean density. All haloes are found to possess a distinct inner core fitted extremely well by the soliton solution (solid lines). A detailed soliton fit for the largest halo is inset, where the error is the rootmean-square scatter of density in each radial bin. A Navarro-Frenk-White (NFW) profile representing standard CDM is also shown for comparison (black dot-dashed line, with a very large scale radius of $10 \mathrm{kpc}$ ), which fits well the profiles outside the cores. The yellow hatched area indicates the $\rho_{300}$ of the dSph satellites around the Milky Way ${ }^{3,24}$, which is consistent with the majority of galaxy haloes formed in the $\psi \mathrm{DM}$ simulations.

(NFW) profile ${ }^{23}$ of standard CDM. These solitonic cores, which are gravitationally self-bound and appear as additional mass clumps superposed on the NFW profile, are clearly distinct from the cores formed by WDM and collisional CDM, which truncate the NFW cuspy inner profile at lower values and require an external halo for confinement. The radius of the soliton scales inversely with mass, such that the widest cores are the least massive and are hosted by the least massive galaxies. Eighty percent of the haloes in the simulation have an average density within $300 \mathrm{pc}$ (defined as $\rho_{300}$ ) in the range $5.3 \times 10^{-3}-6.1 \times 10^{-1} M_{\odot} / \mathrm{pc}^{3}$, consistent with the dSph satellites around the Milky Way ${ }^{3,24}$, and objects like these are resilient to close interaction with massive galaxies. By contrast, the very lowest mass objects in our simulation have $\rho_{300} \sim 4.0 \times 10^{-4} M_{\odot} / \mathrm{pc}^{3}$ and $M_{\text {vir }} \sim 10^{8} M_{\odot}$, but exist only briefly as they are vulnerable to tidal disruption by large galaxies in our simulations. Together with the cutoff in the power spectrum at the Jeans scale (Supplementary Fig. 3), this leads to a marked suppression of substructure below a few times $10^{8} M_{\odot}$ relative to the prediction of standard CDM (refs 8,9). A quantitative evaluation of the mass function of satellite galaxies predicted by $\psi \mathrm{DM}$ with larger simulations is thus another crucial test to be addressed.

The prominent solitonic cores uncovered in our simulations provide an opportunity to estimate the boson mass, $m_{\mathrm{B}}$, by comparison with observations, particularly for $\mathrm{dSph}$ galaxies where dark matter dominates. The local Fornax dSph galaxy is the best studied case, with thousands of stellar velocity measurements, allowing a detailed comparison with our soliton mass profile. We perform a Jeans analysis for the dominant intermediate metallicity stellar population, which exhibits a nearly uniform projected velocity dispersion $\left(\sigma_{\|} ;\right.$ref. 25$)$. We simultaneously 


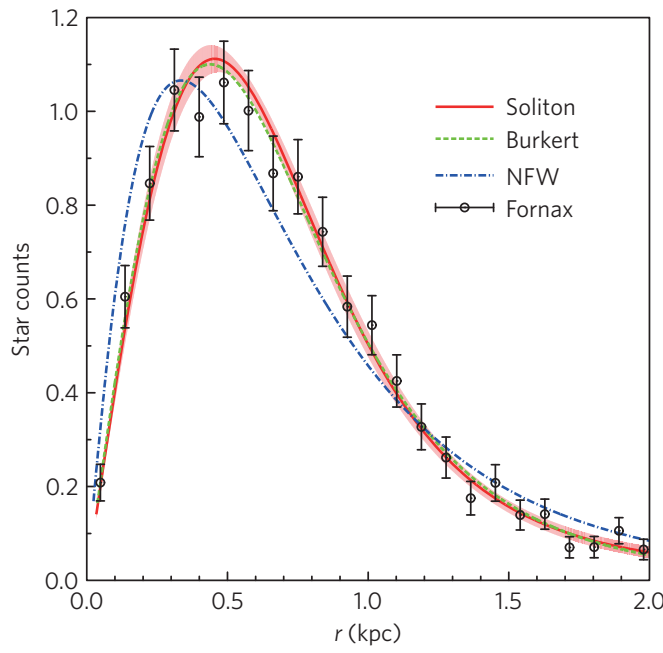

b

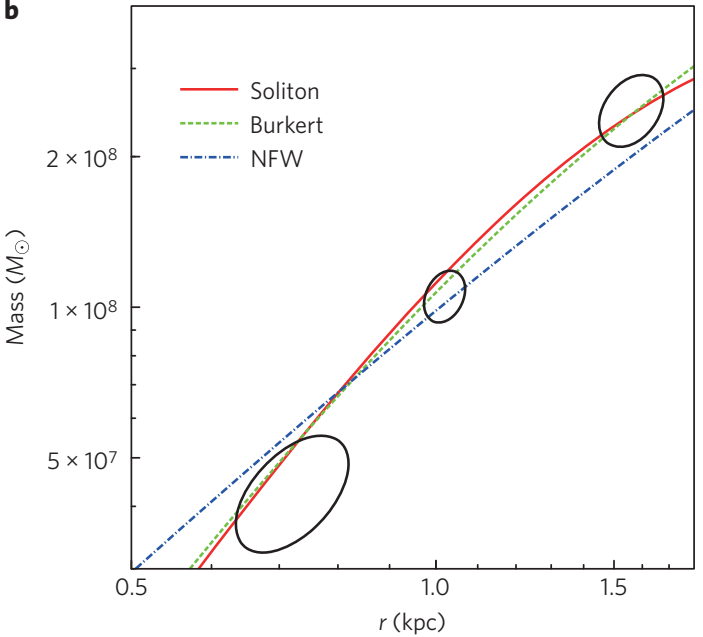

Figure 4 | Modelling the Fornax dSph galaxy with the soliton profile. a, Star counts of the intermediate metallicity subpopulation ${ }^{25}$ at different radial bins (symbols with $1-\sigma$ error bars) and the best-fit soliton solution (red solid line) with $m_{B}=8.0 \times 10^{-23} \mathrm{eV}, r_{\mathrm{C}}=0.93 \mathrm{kpc}$ and $\sigma_{\|}=11.3 \mathrm{~km} \mathrm{~s}^{-1}$, together with the 1- $\sigma$ variation (red shaded). Star counts are normalized to the total number of sample stars within $\sim 2.1 \mathrm{kpc}$. Also shown are the best-fit empirical formula of Burkert ${ }^{35}$ (green dashed line) and the NFW profile (blue dot-dashed line) representing standard CDM. The scale radius of NFW is restricted to be no larger than $3.0 \mathrm{kpc}$ during the fit to exclude unreasonably small concentration parameters. $\mathbf{b}, 1-\sigma$ contours of the total enclosed mass estimated from each of the three subpopulations ${ }^{5}$ (ovals), overplotted with the model curves using the same best-fit parameters adopted in $\mathbf{a}$. Clearly, in both panels the soliton profile of $\psi D M$ provides an accurate fit, matched only by the empirical fitting function of the Burkert profile, whereas NFW is not favoured by the data.

reproduce well the radial distribution of the $\operatorname{stars}^{25}$ (Fig. 4a) and their velocity dispersion with negligible velocity anisotropy, with $m_{\mathrm{B}}=\left(8.0_{-2.0}^{+1.8}\right) \times 10^{-23} \mathrm{eV}$ and a core radius $r_{\mathrm{c}}=0.93_{-0.12}^{+0.19} \mathrm{kpc}$ (Supplementary Fig. 5). The corresponding core mass $M\left(r \leq r_{\mathrm{c}}\right)$ is $\simeq 9.2 \times 10^{7} M_{\odot}$, which is hosted by a halo with virial mass $\simeq 4 \times 10^{9} M_{\odot}$ in the simulations. These results are similar to other estimates for Fornax ${ }^{5,26,27}$ (Fig. 4b) and consistent with other dSph galaxies derived by a variety of means ${ }^{4,26,28}$ (see Supplementary Section 3 for details).

For more massive galaxies, the solitons we predict are denser and more massive, scaling approximately as $M_{\mathrm{s}} \propto M_{\mathrm{vir}}^{1 / 3}$. So for the Milky Way, adopting a total mass of $M_{\mathrm{vir}}=10^{12} \widetilde{M_{\odot}}$, we expect a soliton of
$M_{\mathrm{s}} \simeq 2 \times 10^{9} M_{\odot}$, with a core radius $\simeq 180 \mathrm{pc}$ and a potential depth corresponding to a line-of-sight velocity dispersion $\sigma_{\|} \simeq 115 \mathrm{~km} \mathrm{~s}^{-1}$ for test particles satisfying the virial condition with the soliton potential. At face value this seems consistent with the Milky Way bulge velocity dispersion, where a distinctive flat peak is observed at a level of $\sigma_{\|} \simeq 110 \mathrm{~km} \mathrm{~s}^{-1}$ within a projected radius $\sim 200 \mathrm{pc}$ (refs 29,30). Such cores clearly have implications for the creation of spheroids, acting as an essential seed for the prompt attraction of gas within a deepened potential. Indeed, bulge stars with $[\mathrm{Fe} / \mathrm{H}]>-1.0$ are firmly established as a uniformly old population that formed rapidly $^{30,31}$, a conclusion that standard $\Lambda C D M$ struggles to explain through extended accretion and merging ${ }^{30}$. The implications for early spheroid formation and compact nuclear objects in general can be explored self-consistently with the addition of baryons to the $\psi \mathrm{DM}$ code, to model the interplay among stars, gas and $\psi \mathrm{DM}$, which will provide model rotation curves for an important test of this model.

At high redshift, the earliest galaxies formed from $\psi \mathrm{DM}$ are delayed relative to standard CDM, limited by the small amplitude of the Jeans mass at radiation-matter equality, after which the first structures grow. This is demonstrated with a $\psi \mathrm{DM}$ simulation of a $30 \mathrm{~h}^{-1} \mathrm{Mpc}$ box where we adopt $m_{\mathrm{B}}=8.0 \times 10^{-23} \mathrm{eV}$ derived above. The first bound object collapses at $z \simeq 13$, with a clear solitonic core of mass $\simeq 10^{9} M_{\odot}$ and radius $\simeq 300 \mathrm{pc}$, whereas under $\Lambda \mathrm{CDM}$ the first objects should form at $z \simeq 50$ with masses of only $10^{4}-10^{5} M_{\odot}$ (ref. 32). The highest redshift galaxy at present at $z \simeq 10.7$ is multiply lensed, seeming smooth and spherical, with a stellar radius $\simeq 100 \mathrm{pc}$ (ref. 33), similar to local dSph galaxies. Deeper cluster lensing data from the Hubble 'Frontier Fields' programme will soon meaningfully explore the mass limits of galaxy formation to higher redshift, allowing us to better distinguish between particle and wavelike cold dark matter.

Received 31 August 2013; accepted 15 May 2014; published online 22 June 2014

\section{References}

1. Moore, B. Evidence against dissipation-less dark matter from observations of galaxy haloes. Nature 370, 629-631 (1994).

2. Gilmore, G. et al. The observed properties of dark matter on small spatial scales. Astrophys. J. 663, 948-959 (2007).

3. Strigari, L. E. et al. A common mass scale for satellite galaxies of the Milky Way. Nature 454, 1096-1097 (2008).

4. Walker, M. G. \& Peñarrubia, J. A method for measuring (slopes of) the mass profiles of dwarf spheroidal galaxies. Astrophys. J. 742, 20-38 (2011).

5. Amorisco, N. C., Agnello, A. \& Evans, N. W. The core size of the Fornax dwarf spheroidal. Mon. Not. R. Astron. Soc. 429, L89-L93 (2013).

6. Dubinski, J. \& Carlberg, R. G. The structure of cold dark matter halos. Astrophys. J. 378, 496-503 (1991).

7. Kauffmann, G., White, S. D. M. \& Guiderdoni, B. The formation and evolution of galaxies within merging dark matter haloes. Mon. Not. R. Astron. Soc. 264, 201-218 (1993).

8. Klypin, A., Kravtsov, A. V., Valenzuela, O. \& Prada, F. Where are the missing galactic satellites? Astrophys. J. 522, 82-92 (1999).

9. Moore, B. et al. Dark matter substructure within galactic halos. Astrophys. J. 524, L19-L22 (1999).

10. Peebles, P. J. E. Fluid dark matter. Astrophys. J. 534, L127-L129 (2000).

11. Hu, W., Barkana, R. \& Gruzinov, A. Fuzzy cold dark matter: The wave properties of ultralight particles. Phys. Rev. Lett. 85, 1158-1161 (2000).

12. Marsh, D. J. E. \& Silk, J. A model for halo formation with axion mixed dark matter. Mon. Not. R. Astron. Soc. 437, 2652-2663 (2014).

13. Akerib, D. S. et al. First results from the LUX dark matter experiment at the Sanford Underground Research Facility. Phys. Rev. Lett. 112, 091303 (2014).

14. Peebles, P. J. E. \& Ratra, B. Cosmology with a time-variable cosmological 'constant'. Astrophys. J. 325, L17-L20 (1988).

15. Arvanitaki, A., Dimopoulos, S., Dubovsky, S., Kaloper, N. \& March-Russell, J. String axiverse. Phys. Rev. D 81, 123530 (2010).

16. Widrow, L. M. \& Kaiser, N. Using the Schroedinger equation to simulate collisionless matter. Astrophys. J. 416, L71-L74 (1993). 
17. Woo, T-P. \& Chiueh, T. High-resolution simulation on structure formation with extremely light bosonic dark matter. Astrophys. J. 697, 850-861 (2009)

18. Spergel, D. N. \& Steinhardt, P. J. Observational evidence for self-interacting cold dark matter. Phys. Rev. Lett. 84, 3760-3763 (2000).

19. Bode, P., Ostriker, J. P. \& Turok, N. Halo formation in warm dark matter models. Astrophys. J. 556, 93-107 (2001).

20. Macciò, A. V., Paduroiu, S., Anderhalden, D., Schneider, A. \& Moore, B. Cores in warm dark matter haloes: A Catch 22 problem. Mon. Not. R. Astron. Soc. 424, 1105-1112 (2012).

21. Rocha, M. et al. Cosmological simulations with self-interacting dark matter-I. Constant-density cores and substructure. Mon. Not. R. Astron. Soc. 430, 81-104 (2013).

22. Schive, H-Y., Tsai, Y-C. \& Chiueh, T. GAMER: A graphic processing unit accelerated adaptive-mesh-refinement code for astrophysics. Astrophys. J. Suppl. 186, 457-484 (2010).

23. Navarro, J. F., Frenk, C. S. \& White, S. D. M. The structure of cold dark matter halos. Astrophys. J. 462, 563-575 (1996).

24. Amorisco, N. C. \& Evans, N. W. Phase-space models of the dwarf spheroidals. Mon. Not. R. Astron. Soc. 411, 2118-2136 (2011).

25. Amorisco, N. C. \& Evans, N. W. A troublesome past: Chemodynamics of the Fornax dwarf spheroidal. Astrophys. J. 756, L2-L6 (2012).

26. Wolf, J. et al. Accurate masses for dispersion-supported galaxies. Mon. Not. R Astron. Soc. 406, 1220-1237 (2010).

27. Cole, D. R., Dehnen, W., Read, J. I. \& Wilkinson, M. I. The mass distribution of the Fornax dSph: Constraints from its globular cluster distribution. Mon. Not. R. Astron. Soc. 426, 601-613 (2012)

28. Lora, V., Magaña, J., Bernal, A., Sánchez-Salcedo, F. J. \& Grebel, E. K. On the mass of ultra-light bosonic dark matter from galactic dynamics. J. Cosmol. Astropart. Phys. 2, 11-32 (2012).

29. Minniti, D. Field stars and clusters of the galactic bulge: Implications for galaxy formation. Astrophys. J. 459, 175-180 (1996).

30. Ness, M. et al. ARGOS - IV. The kinematics of the Milky Way bulge. Mon. Not. R. Astron. Soc. 432, 2092-2103 (2013).
31. Zoccali, M. et al. Age and metallicity distribution of the galactic bulge from extensive optical and near-IR stellar photometry. Astron. Astrophys. 399, 931-956 (2003)

32. Abel, T., Bryan, G. L. \& Norman, M. L. The formation of the first star in the universe. Science 295, 93-98 (2002).

33. Coe, D. et al. CLASH: Three strongly lensed images of a candidate $z \approx 11$ galaxy. Astrophys. J. 762, 32-52 (2013)

34. Springel, V. The cosmological simulation code GADGET-2. Mon. Not. R. Astron. Soc. 364, 1105-1134 (2005).

35. Burkert, A. The structure of dark matter halos in dwarf galaxies. Astrophys. J. 447, L25-L28 (1995)

\section{Acknowledgements}

We thank T-P. Woo for calculating the soliton solution and M-H. Liao for helping conduct the simulations. We acknowledge Chipbond Technology Corporation for donating the GPU cluster with which this work was conducted. This work is supported in part by the National Science Council of Taiwan under grants

NSC100-2112-M-002-018-MY3 and NSC99-2112-M-002-009-MY3.

\section{Author contributions}

Each author has contributed significantly to this paper. In particular, T.C. conceived and supervised the project, H-Y.S. developed the code and conducted the simulations, the results of which have been linked by T.B. to the observations.

\section{Additional information}

Supplementary information is available in the online version of the paper. Reprints and permissions information is available online at www.nature.com/reprints.

Correspondence and requests for materials should be addressed to T.C.

\section{Competing financial interests}

The authors declare no competing financial interests. 